\title{
O Perfil dos Fisioterapeutas Egressos da Faculdade de Medicina da Universidade de São Paulo
}

\author{
Sílvia Regina Shiwa ${ }^{1,2}$, Silvia Maria Amado João ${ }^{3 *}$ \\ ${ }^{1}$ Universidade de Guarulhos \\ 2Programa de Pós-Graduação em Ciências da Reabilitação da Faculdade de Medicina da Universidade de São Paulo \\ ${ }^{3}$ Departamento de Fisioterapia, Fonoaudiologia e Terapia Ocupacional da Faculdade de Medicina da Universidade \\ de São Paulo
}

* Autor para correspondência: smaj@usp.br

\section{RESUMO}

O curso de Fisioterapia da Faculdade de Medicina da Universidade de São Paulo (FMUSP) foi o pioneiro no país. Conhecer o perfil do egresso é uma exigência no processo de avaliação institucional, orienta as instituições, embasa políticas públicas e é útil para a sociedade e estudantes como uma referência de cursos e instituições. Porém, não há na literatura informações sobre o perfil dos fisioterapeutas egressos da FMUSP. O presente estudo tem como objetivo descrever o perfil destes, tendo em vista aspectos demográficos, de formação e qualificação profissional, atuação no mercado de trabalho e conhecimento da legislação vigente. Participaram desta pesquisa 110 alunos egressos da FMUSP do curso de Fisioterapia através de um questionário on-line enviado pelo conselho regional da categoria (Crefito-3). Dos fisioterapeutas egressos da referida instituição, 88,2\% são mulheres e jovens, 58,2\% cursaram uma pós-graduação lato sensu e 35,5\% uma pós-graduação stricto sensu principalmente para aprimorar os seus conhecimentos. A leitura de artigos científicos é a principal forma de atualização profissional, $80 \%$ dos participantes estão atuando como fisioterapeutas, e 78,1\% possuem a fisioterapia como sua única fonte de renda, ganhando por mês um salário acima de R \$ 3.000,00 (57,3\%). Pode-se concluir que os fisioterapeutas egressos da FMUSP mostram-se diferenciados quanto à atuação no mercado de trabalho, com maior dedicação à docência e à pesquisa, maior atuação nas universidades, melhores salários e menores dificuldades em se inserirem no mercado de trabalho quando comparados aos fisioterapeutas do estado de São Paulo.

Palavras-Chave: Egresso; Fisioterapia; Avaliação Educacional.

\begin{abstract}
The course of Physiotherapy of the Universidade de São Paulo (USP) was the pioneer one in Brazil. To known the profile of graduates is a requirement in the institutional assessment process, guides the institutions, underlies public policy and it as a reference to society and students about courses and universities. There is no information in the literature about the profile of the graduates of physical therapists of FMUSP yet. This paper will describe the profile of the graduates in physical therapy of USP in accordance with demographics aspects, training and professional qualifications, performance at work and knowledge of the law. We have analyzed 110 graduates students of Physical therapy of USP with online questionnaire that was sent by Regional Council of Physical Therapy and Occupational Therapy (Crefito-3). Of the graduates in physical therapy of USP, $88.2 \%$ are women and young people, $58.2 \%$ did lato sensu post-graduation (master or doctor degree) and 35,5\% did stricto sensu to improve their knowledge. The reading of scientific articles was the main form of professional updates, $80 \%$ of participants was working as physical therapists, $78,1 \%$ had physical therapy as their only source of income and earning monthly salary above $\mathrm{R} \$ 3,000.00$ $(57,3 \%)$. Few professionals participate in associations $(20,9 \%)$ and only $13,6 \%$ of participants were able to inform the correct value of the current minimum wage. It can be concluded that the physical therapist graduates of USP show up as different to other graduates from São Paulo in performance at work, with greater dedication to teaching and research, focused effort in universities, higher wages and lower difficulties to get a job.
\end{abstract}

Keywords: Graduates; Physical Therapy; Educational Assess.

\section{Introdução}

O curso de Fisioterapia da Faculdade de Medicina da Universidade de São Paulo
(FMUSP) foi pioneiro no Brasil, criado em 1951 pelo Dr. Waldo Rolim de Moraes com o patrocínio do centro de estudos Raphael Barros, sendo, 
na época, um curso de nível técnico (MARQUES \& SANCHES, 1994; TRIGO DE SOUZA et al., 2008). Em 1958, iniciou-se o primeiro curso técnico de Fisioterapia do Brasil com padrão internacional mínimo, com duração de dois anos, e, em 1969, a fisioterapia foi regulamentada como profissão e curso de ensino superior através do Decreto Lei n. 938 de 13 de outubro de 1969 (BRASIL, 1969). O curso de Fisioterapia da FMUSP permaneceu com três anos de duração até 1979 e, em 1980, ganhou mais um ano (MARQUES \& SANCHES, 1994; TRIGO DE SOUZA et al., 2008).

Apesar das mudanças na carga horária e da grande expansão do campo de atuação do fisioterapeuta, o currículo de 1963 permaneceu até os anos 1980, acumulando distorções entre o currículo existente e o ministrado pelas faculdades. Devido a isso, em 1983 foram fixados pelo MEG um currículo mínimo e a duração do curso de fisioterapia em 3240 horas. Em 19 de fevereiro 2002, foram instituídas as Diretrizes Curriculares do curso de graduação em Fisioterapia (BRASIL, 2002) para definir princípios, fundamentos, condições e procedimentos de formação de fisioterapeutas, estabelecidos pela Câmara da Educação Superior do Conselho Nacional de Educação, para aplicação em âmbito nacional na organização, desenvolvimento e avaliação dos projetos pedagógicos dos cursos de graduação em Fisioterapia das Instituições do Ensino Superior (IES).

Desde 2004, a avaliação educacional de todos os cursos de nível superior, inclusive a graduação em Fisioterapia, passou a ser realizada pelo Sistema Nacional de Avaliação da Educação Superior (Sinaes), instituído pelo Ministério da Educação e Cultura (MEG) (INEP, 2012). Os processos avaliativos do Sinaes são coordenados e supervisionados pela Comissão Nacional de Avaliação da Educação Superior (Conaes), e a operacionalização é de responsabilidade do Instituto Nacional de Estudos e Pesquisas Educacionais Anísio Teixeira (Inep) (Idem, ibidem). As informações obtidas são utilizadas para a orientação institucional do estabelecimento de ensino superior e para embasar políticas públicas, além de serem úteis para 100 a sociedade, especialmente para os estudantes, como referência quanto às condições de cursos e instituições (Idem, ibidem).

Dentre as dez dimensões avaliadas pelo Sinaes, uma delas faz referência à "política de atendimento a estudantes e egressos", sendo orientado que se observe na instituição de ensino superior se existem dados e indicadores para avaliar esta dimensão, como pesquisas ou estudos sobre os egressos e/ou empregadores dos mesmos. Apesar de esse aspecto ser mandatório para todos os cursos de graduação no Brasil, não existem dados de pesquisa disponíveis sobre qual seria o perfil real dos egressos do curso de fisioterapia da FMUSP (Idem, ibidem).

Além de atender exigências legais, a implantação de um sistema de acompanhamento dos egressos irá oferecer às IES informações relevantes para uma análise autocrítica quanto ao cumprimento da missão da universidade. Com esse acompanhamento dos egressos, será possível avaliar os aspectos relacionados à inserção deles no mercado de trabalho, adequar o currículo do curso de acordo com as exigências do mercado e também propor uma relação mais estreita entre a instituição e o aluno egresso, possibilitando a troca de informações profissionais (empregos, contato com empresas etc.) e acadêmicas (cursos, palestras, simpósios etc.).

Este estudo tem como objetivo descrever o perfil do egresso do curso de Fisioterapia da FMUSP, de acordo com os aspectos demográfico, de formação e qualificação, vínculo técnico-científico, atuação no mercado de trabalho e conhecimento da legislação vigente.

\section{Métodos}

Este estudo utilizou a amostra obtida na pesquisa realizada para identificar o perfil do fisioterapeuta do estado de São Paulo e foi aprovado pelo comitê de ética em pesquisa da Faculdade de Medicina da Universidade de São Paulo (protocolo 152/13 de 24 de abril de 2013).

A distribuição dos convites para participar da pesquisa foi feita pelo Conselho Regional de 
Fisioterapia e Terapia Ocupacional da $3^{\mathrm{a}}$ Região (Crefito-3) via correio eletrônico. Nesse convite constava o link de acesso ao questionário juntamente com o Termo de Consentimento Livre e Esclarecido (TCLE). O questionário utilizado foi criado pelos pesquisadores deste estudo com base nos estudos realizados em outra universidade (UNIVERSIDADE ESTADUAL DE LONDRINA, 2006), sendo excluídas as perguntas irrelevantes. Além disso, tal documento foi composto por 31 questões, com 26 de múltipla escolha e 5 questões abertas. Foi também utilizada uma sequência lógica de respostas, evitando que o participante respondesse perguntas desnecessárias.

Foram selecionados todos os fisioterapeutas graduados na FMUSP, independente do ano de conclusão do curso, com concordância ao TCLE e com respostas completas no questionário aplicado. Paralelamente, foram excluídos os participantes que tinham menos de um ano de graduados.

No que se diz respeito às análises, agruparam-se as variáveis em cinco aspectos (ALTAMIRANDA, 2003): 1) Perfil demográfico: idade e sexo; 2) Formação e qualificação: tipo de instituição, pós-graduação, área da pós-graduação e atualizações na área; 3) Mercado de trabalho: área e local de atuação, distribuição demográfica dos profissionais, regime salarial, satisfação com a profissão, dificuldades na inserção no mercado de trabalho, renda salarial mensal; 4) Vínculo técnico-científico: participação em associações e sindicatos; 5) Conhecimento da legislação vigente: carga horária que compõe a jornada de trabalho e o valor do piso salarial.

Todas as variáveis foram tabuladas em uma planilha do Excel e analisadas com o software IBM SPSS Statistics 21. Foram realizadas análises de estatística descritiva para obter as frequências absolutas e relativas de todos os itens avaliados.

\section{Resultados}

Das 2.323 respostas obtidas somente 110 correspondiam a alunos egressos do curso de fisioterapia da FMUSP. Desta amostra, 97 (88,2\%) eram do sexo feminino, $61,8 \%$ tinham entre 26 e 35 anos e
$79,1 \%$ concluíram o curso de fisioterapia entre os anos de 1991 e 2010 (Tabela 1). Em relação à pósgraduação, 58,2\% cursaram uma pós-graduação lato sensu. As áreas de especialidade mais cursadas foram a acupuntura e a neurofuncional. Porém, a maioria cursou uma especialidade ainda não reconhecida pelo Conselho Federal de Fisioterapia e Terapia Ocupacional (Coffito), sendo a fisiologia do exercício, geriatria e gestão hospitalar as mais citadas. Trinta e nove fisioterapeutas (35,5\%) optaram por realizar uma pós-graduação stricto sensu e possuem como maior titulação o mestrado em $56 \%$ e o doutorado em $41 \%$. Os cursos de extensão (aprimoramento, aperfeiçoamento e cursos de finais de semana) foram feitos por 52\% dos participantes. Constituem os principais motivos alegados pelos participantes para realizarem uma pós-graduação: aprimorar os conhecimentos $(68,2 \%)$, atender a exigência do mercado de trabalho (32,7\%), seguir carreira acadêmica $(27,3 \%)$ (Tabela 2).

Cerca de 80\% dos entrevistados estão atuando como fisioterapeutas, principalmente em atendimentos domiciliares $(43,6 \%)$, hospitais $(23,7 \%)$, consultórios particulares $(18,2 \%)$ e universidades $(15,5 \%)$; consequentemente, o regime salarial predominante é o autônomo. A maioria desses profissionais atua na região da capital paulista e na Grande São Paulo $(78,1 \%)$ e possui a fisioterapia como a única fonte de renda $(69,1 \%)$. Estão inseridos no mercado de trabalho há mais de quatro anos $61,9 \%$, e 45,4\% afirmaram terem encontrado dificuldades na inserção no mercado de trabalho, principalmente devido à forte concorrência. Quanto à renda salarial mensal bruta, 57,3\% afirmam receberem mais de $\mathrm{R} \$ 3.000,00$ por mês, e $72,7 \%$ afirmaram estarem parcialmente satisfeitos a muito satisfeitos (Tabela 3). Somente 20,9\% dos entrevistados participam de algum sindicato ou associação de classe.

As principais formas de atualização utilizadas foram a leitura de artigos científicos $(62,7 \%)$ na frequência de um artigo por semana (34,1\%), cursos de extensão $(43,6 \%)$ e participação em congressos, simpósios e palestras $(58,2 \%)$. Com 


\begin{tabular}{|c|c|}
\hline Sexo & Total (\%) \\
\hline Feminino & $97(88,2)$ \\
\hline Masculino & $13(11,8)$ \\
\hline Idade & Total (\%) \\
\hline Entre 20 e 25 anos & $7(6,4)$ \\
\hline Entre 26 e 30 anos & $37(33,6)$ \\
\hline Entre 31 e 35 anos & $31(28,2)$ \\
\hline Entre 36 e 40 anos & $15(13,6)$ \\
\hline Entre 41 e 45 anos & $9(8,2)$ \\
\hline Entre 46 e 50 anos & $3(2,7)$ \\
\hline Entre 51 e 60 anos & $6(5,5)$ \\
\hline Acima de 60 anos & $2(1,8)$ \\
\hline Ano de conclusão do curso & Total (\%) \\
\hline Entre 1970 a 1980 & $6(5,5)$ \\
\hline Entre 1981 a 1990 & $5(4,5)$ \\
\hline Entre 1991 a 2000 & $26(23,6)$ \\
\hline Entre 2001 a 2010 & $61(55,5)$ \\
\hline Após 2010 & $12(10,9)$ \\
\hline \multicolumn{2}{|c|}{ Tabela 1 - Dados demográficos dos participantes do estudo. } \\
\hline Pós-graduação & Total (\%) \\
\hline Pós-graduação lato sensu & $64(58,2)$ \\
\hline Pós-graduação stricto sensu & $39(35,5)$ \\
\hline Curso de extensão/aprimoramento & $52(47,3)$ \\
\hline Área Pós-graduação lato sensu & Total (\%) \\
\hline Outra & $25(22,7)$ \\
\hline Acupuntura & $15(13,6)$ \\
\hline Neurofuncional & $13(11,8)$ \\
\hline
\end{tabular}




\begin{tabular}{|l|r|}
\hline Cardiorrespiratória & $9(8,2)$ \\
\hline Traumato-ortopédica & $9(8,2)$ \\
\hline Esportiva & $4(3,6)$ \\
\hline Osteopatia e Quiropraxia & $3(2,7)$ \\
\hline Saúde da Mulher & $2(1,8)$ \\
\hline Dermatofuncional & $2(1,8)$ \\
\hline Saúde Coletiva & $1(0,9)$ \\
\hline Trabalho & $0(0)$ \\
\hline Pós-graduação stricto sensu & Total $(\%)$ \\
\hline Mestrado & $22(20,0)$ \\
\hline Doutorado & $17(15,5)$ \\
\hline Pós-doutorado & $1(0,9)$ \\
\hline Por que a opção da pós-graduação & Total $(\%)$ \\
\hline Aprimorar os conhecimentos & $75(68,2)$ \\
\hline Seguir carreira acadêmica/pesquisa & $30(27,3)$ \\
\hline
\end{tabular}

Tabela 2 - Distribuição das frequências relacionadas à formação e qualificação profissional dos fisioterapeutas egressos da FMUSP.

\begin{tabular}{|l|r|l|r|}
\hline $\begin{array}{l}\text { Está atuando como } \\
\text { fisioterapeuta? }\end{array}$ & Total (\%) & Dificuldades na inserção & Total (\%) \\
\hline Sim & $88(80,0)$ & Sim & $40(36,4)$ \\
\hline Não & $20(18,2)$ & Não & $49(44,5)$ \\
\hline Local de atuação & Total (\%) & Motivos & Total (\%) \\
\hline Atendimento domiciliar & $48(43,6)$ & Forte concorrência & $18(16,4)$ \\
\hline Consultório particular & $20(18,2)$ & Falta de experiência & $8(7,3)$ \\
\hline Hospital público & $17(15,5)$ & Satisfação com a profissão & Total (\%) \\
\hline Universidade/docência & $17(15,5)$ & Muito satisfeito & $24(21,8)$ \\
\hline
\end{tabular}




\begin{tabular}{|l|r|r|r|}
\hline Clínica Própria & $10(9,1)$ & Satisfeito & $23(20,9)$ \\
\hline Clínica Terceirizada & $10(9,1)$ & Satisfeito parcialmente & $33(30,0)$ \\
\hline Hospital Privado & $9(8,2)$ & Insatisfeito & $6(5,5)$ \\
\hline Academia & $3(2,7)$ & Muito insatisfeito & $3(2,7)$ \\
\hline Clube e equipe esportiva & $1(0,9)$ & Renda mensal bruta & Total (\%) \\
\hline Empresa e indústria & $4(3,6)$ & Até R\$1.5000,00 & $2(1,8)$ \\
\hline UBS & $1(0,9)$ & R\$1.501,00-R\$3.000,00 & $21(19,1)$ \\
\hline Outro local & $7(6,4)$ & R\$3.001,00-R\$6.000,00 & $33(30,0)$ \\
\hline Regime salarial & Total (\%) & R\$6.001,00-R\$10.000,00 & $18(16,4)$ \\
\hline CLT & $31(28,2)$ & R\$10.001,00-R\$15.000,00 & $10(9,1)$ \\
\hline Servidor público & $15(13,6)$ & Mais de R\$15.000,00 & $2(1,8)$ \\
\hline Prestador de serviços & $3(2,7)$ & & \\
\hline Autônomo & $52(47,3)$ & & \\
\hline Outro & $6(5,5)$ & & \\
\hline & & &
\end{tabular}

Tabela 3 - Distribuição das frequências relacionadas à atuação no mercado de trabalho dos fisioterapeutas egressos da FMUSP.

relação ao conhecimento da legislação vigente, $79 \%$ souberam informar corretamente a carga horária máxima semanal estabelecida por lei de trinta horas semanais, e 13,6\% informaram corretamente o valor do piso salarial de $\mathrm{R} \$ 2.050,00$ estabelecido para o ano de 2013, ano da coleta dos dados deste estudo.

\section{Discussão}

Nesta amostra, observou-se que a maioria dos fisioterapeutas egressos da FMUSP são mulheres, jovens e com pós-graduação, dados que estão de acordo com outras pesquisas que estudaram o perfil do fisioterapeuta (ISRAEL, 1993; ALTAMIRANDA, 2003; TRELHA et al., 2003; PEREIRA \& ALMEIDA, 2006; UEL, 2006; BISPO JR., 2009; BADARÓ \& GUILHEM, 104
2011; CÂMARA \& SANTOS, 2012; SHIWA et al., 2016). Segundo os dados da nossa pesquisa, o número de alunos egressos da FMUSP que realizaram uma pós-graduação lato sensu foi inferior ao encontrado nos outros estudos: 58,2\% entre os alunos da FMUSP, 87,7\% (SHIWA et al., 2016) e 71,1\% (MALERBI \& CASTRO, 2008) entre os fisioterapeutas do estado de São Paulo, 68,5\% entre os egressos da Universidade Federal de Minas Gerais (UFMG) (CÂAMARA \& SANTOS, 2012); porém, o número de alunos com pós-graduação stricto sensu foi superior entre os egressos da FMUSP (35,5\%), quando comparado ao perfil geral do fisioterapeuta do estado de São Paulo (18,9\%) (SHIWA et al., 2016), de Santa Catarina (6,8\%) (ALTAMIRANDA, 2003) e aos egressos da UFMG (24,8\%) (CÂMARA \& 
SANTOS, 2012), sugerindo um maior interesse para a área acadêmica, de docência e pesquisa. Assim, o número de profissionais com atuação nas universidades é maior entre os egressos da FMUSP, proporcionalmente.

Os fisioterapeutas egressos da FMUSP afirmaram ter encontrado dificuldades na inserção no mercado de trabalho em 36,4\% dos casos, devido principalmente à alta concorrência $(16,4 \%)$. Esses valores são inferiores aos afirmados pelos fisioterapeutas do estado de São Paulo, incluindo os egressos da FMUSP: 52,1\% tiveram dificuldades em conseguir o primeiro emprego por causa da falta de experiência $(18,1 \%)$ e da alta concorrência $(27,1 \%)$. Isso mostra que o aluno graduado pela FMUSP se sente mais preparado e encontra menos dificuldade em se inserir no mercado de trabalho. Quanto à renda salarial mensal, o fisioterapeuta egresso da FMUSP tem um salário maior do que os demais fisioterapeutas: 27,3\% recebe mais de $\mathrm{R} \$ 6.000,00$ por mês, comparados aos 9,8\% dos fisioterapeutas do estado de São Paulo (SHIWA et al., 2016).

Em relação ao local de trabalho, os fisioterapeutas egressos da FMUSP apresentaram taxas maiores de atuação em hospitais públicos (4\% a mais), nas universidades (7\% a mais) e em consultórios particulares (5\% a mais) e menor participação em clínicas terceirizadas (4\% a menos), em comparação com os fisioterapeutas do estado de São Paulo (SHIWA et al., 2016). Esses dados demonstram que, devido à sua formação, o incentivo à pesquisa, o relacionamento interpessoal desenvolvido nos campos de estágio e o aproveitamento das oportunidades, eles obtiveram um índice maior de atuação profissional nesses locais de trabalho, e que a minoria está atuando em clínicas terceirizadas: preferem o consultório próprio, buscando a sua autonomia.

As formas de atualização buscadas pelos egressos da FMUSP foram as mesmas encontradas entre os fisioterapeutas do estado de São Paulo (SHIWA et al., 2016), porém os fisioterapeutas egressos da FMUSP participam mais de congressos, cursos e leem mais artigos científicos. Esses valores foram também acima do encontrado no I Censo da Fisioterapia e Terapia Ocupacional realizado pelo Crefito-3 (MALERBI \& CASTRO, 2008) e entre os alunos egressos da Universidade Federal de Minas Gerais (UFMG) (CÂMARA \& SANTOS, 2012).

Em relação à satisfação profissional, os profissionais graduados na FMUSP (72,2\%) estão satisfeitos tanto quanto os egressos da UFMG $(85,8 \%)$ e da Udesc $(65,1 \%)$. Comparados aos fisioterapeutas do estado de São Paulo, os egressos da FMUSP afirmam estarem mais satisfeitos do que os demais, sendo que 21,8\% afirmaram estarem muito satisfeitos, comparados aos 9,6\% dos fisioterapeutas do estado de São Paulo. E o grau de insatisfação também foi menor entre os alunos egressos da FMUSP: 8,2\%, comparados aos $17,8 \%$ dos fisioterapeutas do estado. O nível da satisfação está vinculado à remuneração, e foi observado que os egressos da FMUSP são melhor remunerados do que os demais fisioterapeutas, proporcionalmente.

Entre os fisioterapeutas do estado de São Paulo e os alunos egressos da FMUSP, todos apresentaram baixas taxas em relação ao conhecimento da legislação vigente, como o piso salarial e a carga horária máxima permitida por lei para a profissão. Deve ser enfatizada a importância do conhecimento da legislação desde a graduação; inclusive há a tabela de honorários fisioterapêuticos estabelecida pelo Conselho Federal de Fisioterapia e Terapia Ocupacional (Coffito) para equiparar os valores dos atendimentos, impedindo a concorrência desleal. A legislação deve nortear a profissão, assim como a participação em sindicatos e associação, que lutam por valorizar a profissão.

Os dados apresentados neste estudo mostram que os egressos da FMUSP diferenciam-se dos demais em alguns quesitos; contudo há a necessidade de realizar uma comparação mais abrangente, inclusive entre as universidades públicas e privadas de modo a se considerar a regionalidade das universidades. A avaliação do egresso deveria ser realizada por todas as instituições de ensino, sendo analisados o perfil dos alunos e as suas 
opiniões quanto à sua formação e à infraestrutura oferecida. Dessa forma, obter-se-iam informações que poderiam favorecer o crescimento da profissão e do ensino.

\section{Conclusão}

Os fisioterapeutas egressos da FMUSP mostram-se diferenciados quanto à atuação no mercado de trabalho, com maior dedicação à docência e à pesquisa, maior atuação nas universidades, melhores salários e menores dificuldades em se inserir no mercado de trabalho quando comparados aos fisioterapeutas do estado de São Paulo.

\section{Agradecimentos}

Agradecemos ao Crefito-3 pelo apoio neste trabalho.

\section{Referências Bibliográficas}

ALTAMIRANDA, E. E. F. Perfil do Fisioterapeuta no Estado de Santa Catarina. Dissertação (Mestrado em Saúde Pública). Programa de Pós-Graduação em Saúde Pública, Universidade Federal de Santa Catarina, 2003.

BADARÓ, A. F. V. \& GUILHEM, D. "Perfil Sociodemográfico e Profissional de Fisioterapeutas e Origem das suas Concepções sobre Ética". Fisioter. Mov., vol. 24, n. 3, 2011, pp. 445-454.

BISPO JR., J. P. "Formação em Fisioterapia no Brasil: Reflexões sobre a Expansão do Ensino e os Modelos de Formação". História, Ciências, Saúde Manguinhos, vol. 16, n. 3, 2009, pp. 655-668.

BRASIL. Decreto-Lei n. 938 de 13 de outubro de 1969, 1969.

BRASIL. Ministério da Educação. Conselho Nacional de Educação - Câmara de Educação Superior. Resolução CNE/CES 4, 19 fev. 2002. Disponível em: $<$ https://www.abmes.org.br/arquivos/legislacoes/res_ces_ cne_04_190202.pdÐ. Acessado em 02 jul. 2018.

CÂMARA, A. M. C. S. \& SANTOS, L. L. C. P. "Um Estudo com Egressos do Curso de Fisioterapia da Universidade Federal de Minas Gerais (UFMG)
- 1982-2005". Rev Bras Ed Med, vol. 36, n. 1, Supl. 1, 2012, pp. 5-17.

INEP. Sistema Nacional de Avaliação da Educação Superior (SINAES). 2012. Disponível em: <http:// portal.inep.gov.br/superior-sinaes $>$. Acessado em 28 fev. 2012.

ISRAEL, V. L. Caracterização da Atuação Profissional de um Grupo de Fisioterapeutas da Cidade de Curitiba. Dissertação (Mestrado em Educação Especial). Programa de Pós-Graduação em Educação Especial, Universidade Federal de São Carlos, São Carlos, 1993.

MALERBI, F. E. K. \& CASTRO, Y. "Análise dos Dados Obtidos no I Censo dos Fisioterapeutas e Terapeutas Ocupacionais do Estado de São Paulo". Crefito, vol. 3, 2008, 41 p.

MARQUES, A. P. \& SANCHES, E. "Origens e Evolução da Fisioterapia: Aspectos Históricos e Legais". Rev Fisioter Univ São Paulo, vol. 1, n. 1, 1994, pp. 5-10.

PEREIRA, L. A. \& ALMEIDA, M.J. "Fisioterapia". In: MINISTÉRIO DA SAÚDE E FUNDAÇÃO OSWALDO CRUZ (Eds.). Dinâmica das Graduações em Saúde no Brasl: Subsídios para uma Política de Recursos Humanos. Brasília: Editora MS-OS 2006/0291, 2006, pp. 171-184. Série G. Estatística e Informação em Saúde.

SHIWA, S. R.; SCHMITT, A. C. B. \& JOÃO, S. M. A. "O Fisioterapeuta do Estado de São Paulo". Fisioter. Pesq., vol. 23, n. 3, 2016, pp. 301-10.

TRELHA, C. S.; GUTIERREZ, P. R. \& GUNHA, A. C. V. "Perfil Demográfico dos Fisioterapeutas da Cidade de Londrina/PR”. Salusvita, vol. 22, n. 2, 2003, pp. 247-256.

TRIGO DE SOUZA, L. M.; MOTA, A. \& MARINHO, Maria Gabriela S. M. G. FOFITO - 50 anos de Pioneirismo e Lutas. São Paulo: Departamento de Fisioterapia, Fonoaudiologia e Terapia Ocupacional da FMUSP, 2008.

UNIVERSIDADE ESTADUAL DE LONDRINA (UEL). Acompanhamento do Egresso. Coordenação de Ricardo de Jesus Silveira. Londrina: UEL, 2006, 65p. (Cadernos de avaliação institucional, 5).

UNIVERSIDADE ESTADUAL DE LONDRINA. Acompanhamento do Egresso. Pró-Reitoria de Planejamento. Londrina: UEL, 2006.

Publicado em 04/07/2018. 